\title{
Speech Database Compacted for an Embedded Mandarin TTS System
}

\author{
Qing Guo, Bin Wang \\ Internet Application Laboratory \\ Fujitsu Research and Develop Center China \\ Beijing, P.R.C \\ guoqing@cn.fujitsu.com
}

\author{
Nobuyuki Katae \\ Audio-Visual Systems Laboratory \\ Fujitsu Laboratories Ltd. \\ Akashi, Japan \\ katae@jp.fujitsu.com
}

\begin{abstract}
In recent years, the unit selection based concatenative speech synthesis system that uses large speech database has become popular because it can produce high quality synthesized speech. However, using such a large speech database is not practical for many applications such as those ported on embedded devices with the storage requirement and the computational complexity involved in searching it. In this paper, it proposed the context based pruning algorithm and waveform adjustment effect based pruning algorithm to compact the speech database. At last, it presents experimental results and discussion.
\end{abstract}

Keywords-text-to-speech; segment pruning;prosody structure; unit selection

\section{INTRODUCTION}

A Text-To-Speech (TTS) synthesizer is a computer-based system that can read text aloud automatically, regardless whether the text is introduced by computer input stream or a scanned input submitted to an optical character recognition (OCR) engine. TTS synthesis can be used in many areas, such as telecommunication services, language education, vocal monitoring, multimedia, and it can also be used as an aid to handicapped people. Impressive progress has been made over the last couple of decades in Mandarin TTS research. A lot of high quality Mandarin TTS systems have become mature in commercial use.

Concatenative speech synthesis that uses large speech database has become popular in recent years due to their improved sensitivity to unit context over their simpler predecessors. These systems usually make use of large speech databases and employ sophisticated search algorithms to determine the optimal unit sequence used to synthesize each sentence.

During synthesis, the input text is converted into a phonetic string with predicted prosodic targets. The synthesis system then searches for speech segments that are close to the context of the target phonetic string and its predicted prosodic targets. At last, a pitch and duration modification algorithm, such as PSOLA, is applied to pre-stored units to guarantee that the prosodic features of synthetic speech meet the predicted target values and the transformed waveforms are concatenated together. Some Mandarin TTS systems adopt two-module TTS structure[1][2]. These systems bypass the prosody module because there is no pitch or duration modification will be applied to the selected units before concatenation.

Since comprehensive linguistic and acoustic phenomena coverage is essential for a high quality concatenative speech synthesis system that the greedy algorithm[3] is often adopted for speech corpus designing to cover more phonetic context and prosodic context phenomena in a recording corpus of a given size. Generally speaking, it's a latest trend that the size of the speech databases recorded in Mandarin TTS systems becomes bigger and bigger.

The size of these speech databases may be as large as several hundred megabytes or even several gigabytes. However, using such a large speech database is not practical for many applications such as those applications ported on embedded devices with the storage requirement and the computational complexity involved in searching it. Thereafter, it is necessary to compact the speech database by some off-line segment pruning algorithms to determine which subset of the database can enable the minimum degradation in synthesis quality to be performed for a given runtime system size.

To improve the efficiency of the speech synthesis systems and maintain the quality of the synthesized speech at the same time, some researchers use some segment pre-selection algorithms to specify a small set of candidate segments from the entire speech database for a target segment. In fact, these segment pruning algorithms and segment pre-selection algorithms often use the same techniques.

Some previous systems reported in the publications have used simple heuristics to select a single version of each unit[4][5], while others have used more complex procedures to select multiple versions[6-10].

Black A.W.[6] adopted a clustering algorithm to cluster units within a unit type based on questions concerning prosodic and phonetic context with an acoustic measure, which uses an acoustic vector comprising of Mel frequency cepstrum coefficients, F0, power and delta cepstrum, F0, power. Donovan R.E.[8] defined a usefulness measurement for each segment. Only those most useful segments are saved for the synthesizer at runtime. Hamza W.[9] proposed a data-driven algorithm to reduce the database size used in concatenative synthesis. The algorithm retains speech segments based on statistics collected by synthesizing a large number of sentences 
using the full speech database. Ling Z.H.[10] introduced an unit pre-selection method by classification and regression tree (CART) for Mandarin synthesis system to improve its efficiency.

Generally speaking, segment pruning algorithm or segment pre-selection algorithm has two problems need solving. One is how to measure the difference between two segments in the clustering process. The other is how to select a representative segment for each cluster.

This paper is organized as follows. Section 2 introduces the FUJITSU Mandarin TTS system briefly, which is a state-ofthe-art unit selection based concatenative speech synthesis system[11]. Section 3 describes two segment pruning algorithms that were used to compact the speech database for embedded TTS system. Section 4 provides the experimental results of listening test. Finally, it makes a conclusion in Section 5.

\section{SYNTHESIS SYSTEM OVERVIEW}

\section{A. Basic Synthesis Unit}

In the Fujitsu Mandarin TTS system, syllables are the basic synthesis units in the unit selection model. However, in order to avoid serious degradation of synthesized speech quality, initials and finals are processed respectively with a PSOLA algorithm to have the speech prosody and concatenating speech waveforms modified.

There are about 205 syllable initials and finals, 1,600 tonal syllables in Mandarin. Using syllable initials and finals as basic units can improve the robustness of a statistic decision tree model. 182 finals and 23 initials have been defined in our system. In addition, some high frequency retroflex finals are also been considered.

In our study, syllable initials and finals (with tone) are defined as the basic synthesis units.

\section{B. Corpus Design}

Speech corpus design is critical in building high quality text for speech synthesis systems. Usually, reading speech is adopted for it seems to be the easiest way to obtain a recorded speech corpus with highest control of the content. Comprehensive linguistic phenomena coverage is essential for a high quality synthesized speech TTS system. Greedy algorithm[3] was adopted for sentence selection to cover more phonetic context and prosodic context phenomena in a recording corpus of a given size.

The text source of our database was the Chinese People Daily 1998 Corpus, which is transcribed from a Chinese newspaper, with word segmentation and POS-tag annotated for natural language processing purpose. Using greedy algorithm, when 3,277 sentences were selected, all 18,985 high frequency vectors are covered. 83 sentences were selected to cover 119 high frequency r-syllables (with retroflex final in it) not covered by those 3,277 sentences. Eventually 3,360 sentences (with about 200k Chinese characters) and 1,550 isolated tonal syllables were recorded in our TTS corpus.

\section{Prosodic Structure Labeling}

The prosody structure is composed of four tiers[12]: prosodic word (PW), minor phrase (MIP), major phrase (MAP) and intonation group (IG). Prosodic word is a tone group bearing one word stress. Minor phrase contains one or more prosodic words, bears one phrasal stress and the perceived break between MIPs is longer than that between PWs. MAP contains one or more PWs, bears one phrasal stress and the perceived break between MAPs is longer than that between MIPs. The criterion for prosody structure labeling is listening perception. Major phrases are often marked by commas with incomplete pitch resetting while intonation groups are marked by periods, quotation marks or semicolons with full pitch resetting.

Additionally, three levels of stress have been defined, namely the stressed, the normal and the neutralized.

The following is a sample transcription of a certain sentence in the speech corpus. "|", “||", “||" and “@” represent PW, MIP, MAP and IG in the transcription respectively. A syllable marked with " $\mathrm{H}$ " means that it is a stressed syllable, and a syllable marked with "L" means that it is a neutralized one.

8 月(bal yve4_H)/t | 20 日 $(\mathrm{er} 4 \mathrm{sh} \% 2$ H r r 04 H $) / \mathrm{t} \mid$ 清晨 (qing1_H chen2)/t , $\| \mid$ - (yi1) $/ \mathrm{m}$ 支 $(\mathrm{zh} \% 1$ 1_H) $/ \mathrm{q}$ 满载(man3 zai4_H) $/ v||$ 锅碗漂盆(guo1_H wan3 piao2_H pen2)/1 、|| 桌 椅(zhuo1_H yi3)/n 、 || 调料(tiao2_H liao4)/n 、 || 发电机(fa1 dian4 ji1_H)/n || 等 $($ deng3) $/ \mathrm{u}$ | 家当 $(\mathrm{jia} 1$ dang4_H) $/ \mathrm{n}$ 的 (de5_L) $/ \mathrm{u}||$ 流动(liu2 dong4_H) $/ \mathrm{vn}$ | 支前 $(\mathrm{zh} \%$ 1_H qian2) $/ \mathrm{vn}$ 车队 (che1_H dui4)/n $\|$ 从 (cong2_H) $/ \mathrm{p}$ 郑州 (zheng4 zhou1_H)/ns $\mid$ 出发(chu1 fa1_H)/v了 (le 5_L $/ \mathrm{y} 。 @$

\section{THE PRUNING ALGORITHMS}

The performance of a pruning algorithm depends on the infrastructure of a TTS system itself. Generally, a TTS system contains three modules; they are the text analysis module, the prosody generation module and the back-end module. The back-end module is responsible for unit selection and speech synthesis. To ensure that the unit selection algorithm can find the most appropriate candidate, a speech database must be diverse enough. The diversity of the speech database means that the syllables in the speech database have many samples with different prosody parameter and acoustic parameter.

To implement an embedded TTS system used for those applications ported on embedded devices, two pruning algorithms are designed to compact the entire speech database in this paper. These two pruning algorithms, context based pruning algorithm and waveform adjustment effect based pruning algorithm, are described in this section respectively.

\section{A. Context based Pruning Algorithm}

A pruning algorithm should have two effects. One is that it can remove spurious atypical units which may have been caused by mislabeling or poor articulation in the original recording. The other one is that it can remove those units which are so common that there is no significant distinction between candidates. 
As for the second type of pruning, removing those overly common units, three factors should be considered. Firstly, the diversity of prosody among segment samples should be maintained. Secondly, the diversity of spectrum among segment samples should be maintained. Thirdly, the diversity of context should be covered as much as possible.

Figure 1 gives the framework of the first pruning algorithm implemented in our Mandarin TTS system.

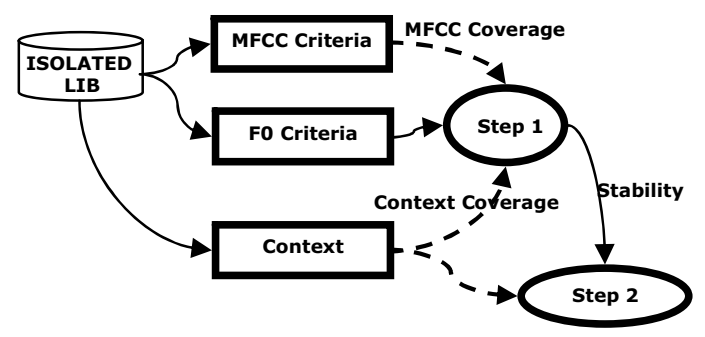

Figure 1. The framework of the context based pruning algorithm.

It can be observed from the figure that there are 2 steps in the first pruning algorithm.

In step 1, two clustering algorithms are implemented, which adopt MFCC parameter and pitch contour to measure the difference between two segments in the clustering process respectively. As we know, the most important indicator of prosody information is pitch contour in Mandarin. The variety of pitch range and pitch pattern is the basis of the continuity of synthesized speech because that too much pitch conversions resulting in bad synthesis voice quality. Therefore, we used pitch contour based clustering algorithm in the listening test, which will be mentioned in the next section. Hence, the syllables which have similar pitch contours are put together in a same cluster. As a result, syllables with variety of pitch contours will be reserved in the compacted speech database.

In addition, considering that the diversity in prosody and acoustic of a syllable is caused by its different context in the speech, a context coverage strategy is used to ensure that those syllables with high frequency context types are reserved in the step 1. The context type of a syllable in the speech database is defined by six factors as following: The category of the previous syllable; The category of the following syllable; The tone of the previous syllable; The tone of the following syllable; Syllable position in the prosodic word that the syllable belongs to; Prosodic word location in the prosodic phrase that the syllable belongs to.

In step 2, a stability pruning strategy is used for furthermore compacting. Using the predicted pitch contour and the predicted duration of a syllable as a reference, a stability measurement can be scored for all the reserved syllables in the step 1 . The context coverage strategy can be used in step 2 too. However, the dimension of the context type of a syllable is usually smaller than that used in step 1 .

\section{B. Waveform AdjustmentEeffect based Pruning Algorithm}

The second pruning algorithm is a clustering algorithm also based on pitch contour. As we know, a pitch and duration modification algorithm, such as PSOLA, is applied to prestored units to guarantee that the prosodic features of synthetic speech meet the predicted target values. Hence, a new representative unit selecting algorithm by considering the effect of waveform adjustment is used in our second pruning algorithm.

Firstly, all samples of a certain syllable are clustered together. To cluster these candidates, it uses their pitch contours to measure the distance between two samples of a certain syllable.

Then, a representative waveform is selected from each cluster. In order to find a good representative unit, a new measurement method is defined to find the most stable unit in the cluster.

Considering that a pitch and duration modification algorithm will be applied to the selected representative unit to satisfy the predicted target pitch and duration, a stable metric of any unit in a cluster is defined as follows:

Let us denote the waveform of the unit A as Waveform-A. Before calculating the distance between the unit and any other unit (herein it refers to as target unit, Waveform-B) in the cluster, Waveform-A is converted into the target pitch contour and duration of the target waveform at first. The converted waveform is denoted as Waveform- $\mathrm{A}^{\prime}$. Then, calculate the low power spectrum distance between Waveform- $\mathrm{A}^{\prime}$ and Waveform-B. The sum of all distances between the unit A and other units in the same cluster is used as the stable metric of the unit A. At last, the unit with lowest stable metric score will be selected as the representative unit of each cluster.

\section{EXPERIMENTAL RESULTS}

Firstly, in order to evaluate the effectiveness of our two pruning algorithms, we conducted paired comparing, in which synthesized speech using the compacted speech databases by two pruning algorithm were compared.

20 sentences were used in the pruning algorithms evaluation. 30 people were asked to make comment on each pair of waveforms, A and B. There were 5 mutually exclusive choices for answers, namely: A is better; $\mathrm{A}$ is a little better; equal; $\mathrm{B}$ is a little better; and, $\mathrm{B}$ is better.

TABLE I. THE PAIRED COMPARING RESULTS

\begin{tabular}{|c|l|}
\hline Wave DB & $\begin{array}{l}\text { Averaged } \\
\text { Score }\end{array}$ \\
\hline 200M (Method 1) vs. 200M (Method 2) & 2.8 vs. 3.2 \\
\hline 50M (Method 1) vs. 50M (Method 2) & 2.9 vs. 3.1 \\
\hline
\end{tabular}

Table 1 gives the results of the pruning algorithms evaluation. To evaluate which pruning algorithm performs well in the evaluation, we marked the above five answers as 5, 4, 3, 2 and 1 respectively. Then, the averaged score was used as the criteria to judge which pruning algorithm is better. 
Experimental results show that the second pruning algorithm is a little better than the first one. Hence, in the next turn of listening tests, we'll use the second pruning algorithm to compact the speech database.

Then we conducted another listening test to evaluate the degradation category rating (DCR) of synthesized speech according to different speech database compacting ratios.

Still 20 sentences were used in the DCR evaluation. 30 people were asked to make comment on each four synthesized waveforms using $1.8 \mathrm{G}$ wave $\mathrm{DB}$ (the full set of our speech database), $200 \mathrm{M}$ wave $\mathrm{DB}, 50 \mathrm{M}$ wave $\mathrm{DB}$ and $2.6 \mathrm{M}$ wave $\mathrm{DB}$ comparing with natural speech of these 20 sentences.

The five answering standards were prepared in the DCR evaluation as following:

5: Inaudible (can not percept the difference between the two data);

4: Audible, but not annoying;

3: Slightly annoying;

2: Annoying;

1: Very annoying.

The listeners made comment on each speech datum. The DCR score was the average score of all listeners.

Remarks. The manual transcribed front-end (linguistic processing) results of 20 sentences were used in both two listening tests.

Figure 2 gives the results of the DCR results of $1.8 \mathrm{G}$, 200M, 50M, 2.6M wave DB respectively.

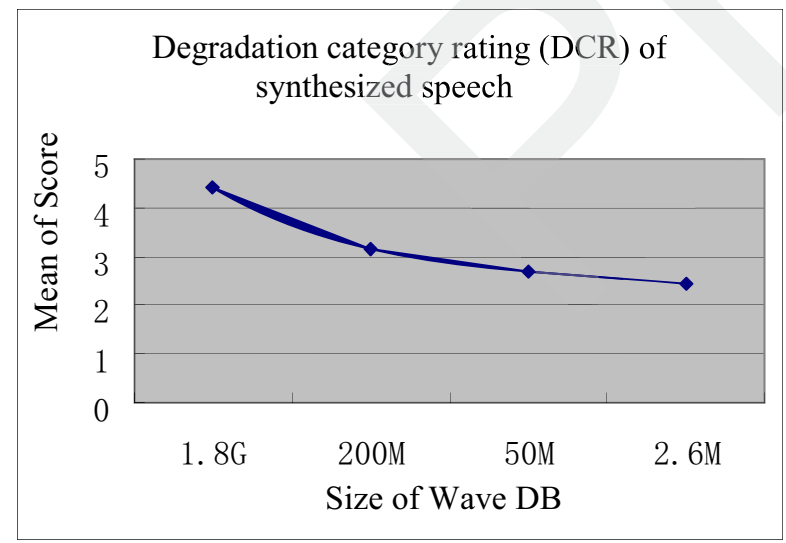

Figure 2. DCR results of the second listening test.

\section{CONClusions}

In this paper, it proposes to use context based pruning algorithm and waveform adjustment effect based pruning algorithm to compact the speech database. The first method emphasized on the coverage of high frequency context types during the compacting process. The second one defined a stable measurement to characterize the effect of waveform adjustment during synthesis of a waveform unit. The most stable unit was selected as representative unit from each cluster to expect that the minimum waveform adjustment degradation was obtained during synthesis. Lastly, it carried out two paired comparing listening tests to evaluate the effectiveness of two pruning algorithms and the degradation category rating (DCR) of synthesized speech according to different speech database compacting ratios.

\section{ACKNOWLEDGEMENT}

The first pruning algorithm described in this paper is developed on the basis of the research work of a collaborative project with Institute of Automation, Chinese Academy of Sciences.

\section{REFERENCES}

[1] Chu M., Peng H., Yang H.Y. and Chang E.., "Selecting non-uniform units from a very large corpus for concatenative speech synthesizer", International Conference on Acoustic, Speech and Signal Processing, Salt Lake City, 2001

[2] Chu M, Peng H. and Chang E., "A concatenative Mandarin TTS system without prosody model and prosody modification", Proceedings of 4th ISCA workshop on speech synthesis, Scotland, 2001.

[3] van Santen, J P. H. and Buchsbaum, A. L., "Methods for optimal text selection ", Proc. of Eurospeech, p. 553-556, Rhodes, Greece, 1997.

[4] Donovan, R.E., andWoodland, P.C. (1999) A Hidden Markov Model Based Trainable Speech Synthesiser, Computer Speech and Language, Vol. 13, No. 3, pp. 223-242.

[5] Itoh, K., Nakajima, S., and Hirokawa, T. (1994) A NewWaveform Speech Synthesis Approach Based on the COC Speech Spectrum, Proc. ICASSP'94, Adelaide, Vol. 1, pp. 577-580.

[6] Black, A.W., and Taylor, P. (1997) Automatically Clustering Similar Units for Unit Selection in Speech Synthesis, Proc. Eurospeech'97, Rhodes., pp. 601-604.

[7] Hon, H., Acero, A., Huang, X., Liu, J., and Plumpe, M. (1998) Automatic Generation of Synthesis Units for Trainable Text-to-Speech Systems, Proc. ICASSP'98, Seattle, pp. 293-296.

[8] Donovan R.E., "Segment pre-selection in decision-tree based speech synthesis systems", International Conference on Acoustic, Speech and Signal Processing, 2000.

[9] Hamza W. and Donovan R.E., "Data-Driven Segment Preselection in the IBM Trainable Speech Synthesis System", International Conference on Spoken Language Processing, 2002

[10] Ling Z.H., Hu Y., Shuang Z.W., Wang R.H., "Decision tree based unit pre-selection in Mandarin Chinese synthesis", International Conference on Spoken Language Processing, 2002.

[11] Guo Q., Katae N., Yu H. and Iwamida H., "High quality prosody generation in a text-to-speech system", Journal of Chinese Information Processing, Vol.22, No 2, 2008.

[12] Li, A. and Lin, M., "Speech corpus of Chinese discourse and the phonetic research", International Conference on Spoken Language Processing, 2000.

[13] Guo Q. and Katae N., "Duration prediction in Mandarin TTS system", 3rd International Conference on Speech Prosody, 2006.

[14] Guo Q., Katae N., Yu H. and Iwamida H., "Decision tree based duration prediction in mandarin TTS system", Journal of Chinese Language and Computing 17 (2): 97-106, 2007.

[15] Guo Q. and Katae N., "Statistical prosody generation in Mandarin TTS system", OCOCOSDA, 2007. 